\title{
Site-controlled quantum emitters in monolayer $\mathrm{MoSe}_{2}$
}

Leo Yu ${ }^{1, *}$, Minda Deng ${ }^{1,4}$, Jingyuan Linda Zhang ${ }^{1,4}$, Sven Borghardt ${ }^{2}$, Beata Kardynal ${ }^{2}$, Jelena Vučković ${ }^{1}$, and Tony F. Heinz ${ }^{1,3, *}$

${ }^{1}$ E. L. Ginzton Laboratory, Stanford University, Stanford, CA 94305, USA

${ }^{2}$ Peter Grünberg Institute, Forschungszentrum Jülich, 52425 Jülich, Germany

${ }^{3}$ SLAC National Accelerator Laboratory, Menlo Park, CA 94025

${ }^{4}$ These authors contributed equally.

*e-mail: leoyu@stanford.edu; tony.heinz@stanford.edu

\section{Supporting Information}

Supporting Information 1. Strain distribution in traps

Supporting Information 2. Additional experimental results on exciton emission for traps of various diameters Supporting Information 3. Spectral wandering of exciton emission

Supporting Information 4. Analysis of $g^{(2)}$ correlation data

Supporting Information 5. Additional QE magneto-optical and polarization measurements

Supporting Information 6. Characteristics of $\mathrm{WSe}_{2}$ quantum emitters

Supporting Information 7. Modeling of exciton-localization effects in traps

Supporting Information 8. Additional contributions towards exciton localization in $\mathrm{MoSe}_{2}$ quantum emitters

Supporting Information 9. Distinguishing between neutral excitons and charged excitons

Supporting Information 10. Radiative rates of localized excitons

Supporting Information 11. Temperature-dependent photoluminescence from $\mathrm{MoSe}_{2}$ quantum emitters 


\section{Supporting Information 1. Strain distribution in traps}

We constructed topography of the milled trap to mimic the shape of a clamped circular membrane deformed under uniform pressure. This problem was first been addressed by Hencky ${ }^{1,2}$, who assumed the membrane does not move at the clamped edge. His solution was later generalized to account for finite clamping friction on the flat part of the surface in the so-called extended Hencky solution ${ }^{3}$. In the following, we present the Hencky solution to illustrate the pressure response of the membrane from the center to the edge. The extended Hencky solution is then summarized and used in our numerical modelling.

Hencky solved the mechanical problem ${ }^{1,2}$ of a clamped circular membrane deformed under uniform pressure using a power series in the radial coordinate $r \leq a$, the membrane radius. The resulting height profile $z(r)$, radial stress $\sigma_{r}$, and circumferential stress $\sigma_{\theta}$ are given by

$$
\begin{aligned}
& z(r)=a q^{1 / 3}\left[\frac{1}{B_{0}}-\frac{1}{B_{0}}(r / a)^{2}+\cdots\right] \\
& \sigma_{r}=\frac{1}{4} E q^{2 / 3}\left[B_{0}-\frac{1}{B_{0}^{2}}(r / a)^{2}+\cdots\right] \\
& \sigma_{\theta}=\frac{1}{4} E q^{2 / 3}\left[B_{0}-\frac{3}{B_{0}^{2}}(r / a)^{2}+\cdots\right]
\end{aligned}
$$

Here $q=(p a / E h)$ is the normalized pressure, where $p$ is the external pressure, $E$ is the Young's modulus of the membrane, and $h$ is the membrane thickness. The factor $B_{0}$ is a function of the Poisson ratio $v$. These equations, together with the usual stress-strain relationships, $\varepsilon_{r}=\left(\sigma_{r}-v \sigma_{\theta}\right) / E$ and $\varepsilon_{\theta}=\left(\sigma_{\theta}-v \sigma_{r}\right) / E$, determine the strain distribution. Note that the strain at the center of the trap is isotropic. Appropriate material parameters for monolayer $\mathrm{MoSe}_{2}$ are $E=159 \mathrm{GPa}^{4,5}$ for a monolayer thickness of $h=0.65 \mathrm{~nm}$ and a Poisson ratio of $v=0.23$.

The Hencky solution shows that for different applied pressures $p$ and trap radii $a$, the shape of $z(r)$ remains the same, only changing in overall scale. To maintain the same maximum strain profile as the radius $a$ of the trap is varied we need to hold the parameter $q$ constant, i.e., varying $p \sim 1 / a$.

While predicting largely correct pressure response of the membrane inside the edge, Hencky's solution requires an infinitely large clamping force at the edge, and thus predicts that a sizable strain ( half of the maximum) at the edge would abruptly drop to zero outside the edge. This artificial behavior is resolved in the extended Hencky's solution by replacing the fixed boundary condition with matched stresses inside and outside the edge ${ }^{3}$. This fully defines the strain in the system as a function of a parameter $q^{2 / 3} / F$, where $F=(f a / E h)$ is a normalized friction parameter and $f$ is the sliding frictional force per unit area. Large normalized pressure causes the same effects as small friction. For the strain distribution around our 50-nm traps where $q^{2 / 3} / F$ reaches several hundreds, the predicted strain at the edge is less than $5 \%$ of the maximum strain, and decreases to zero at $r \sim 8 a$. To exclude the variation in friction due to materials and substrates, we take the strain at the edge to be zero in our numerical modelling in Supporting Information 7.

The Hencky solution, extended or not, predicts a nearly quadratic profile for the height $z(r)$. In Figure S1a, we calculate for the $\mathrm{MoSe}_{2}$ monolayer the predicted height profile $z(r)$ including terms up to $r^{10}$ and compare to the case of only retaining the constant and quadratic terms. The two profiles for each solution, corresponding to a maximal strain of $1.3 \%$, differ by less than $10 \%$. The pressure $p$ to achieve the profiles differs somewhat: $p$ is $\sim 20 \%$ higher when retaining only terms to quadratic order. 

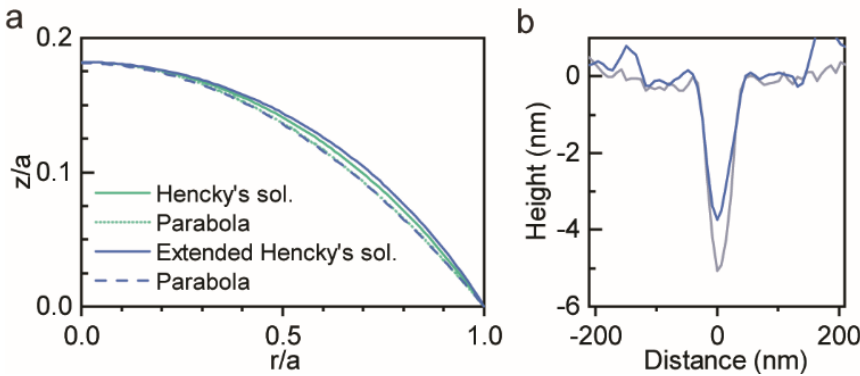

Figure S1. Height profiles of the Hencky solutions. (a) Membrane height profiles $z(r)$ as a function of radial distance $r$ calculated using the Hencky' solution, without and with an extension to finite friction, with radial terms kept up to $r^{10}$ compared with a parabolic profile for the same maximum biaxial strain of $\sim 1.3 \%$. The two profiles in each solution differ by less than $10 \%$. (b) Trap depth profile measured by atomic-force microscopy for the QE2. The figure presents profiles of the bare 50-nm trap prior to dry transfer (grey) and of the trap with the $\mathrm{MoSe}_{2}$ monolayer in place (blue). These data show that the topography variation is approximately parabolic and controlled at the level of a nanometer.

Since the profiles predicted by the Hencky solutions are well approximated by parabolas, we used a fabrication procedure designed to produce a parabolic topography profile in the oxide $\left(\mathrm{SiO}_{2}\right)$ substrates. We utilized a focused ion beam (FIB, FEI Helios NanoLab 600i) whose finite interaction volume with the substrate leads to a minimal topography feature of Gaussian shape and $~ 20-\mathrm{nm}$ full width at half maximum. For the 1000-nm diameter traps (not shown), we scaled the beam dwell time at individual pixels of patterns in proportion to the desired parabolic depth variation. For 50-nm diameter trap, the Gaussian-shaped, 20-nm minimal features produced an approximately parabolic depth variation, as shown in Figure S1b, without varying dwell times as the ion beam was scanned.

For 1- $\mu \mathrm{m}$-diameter parabolic profiles, optical mapping of the photoluminescence revealed results compatible with the predicted spatial strain profile 6 . While we were not able to resolve the spatial distribution for smaller traps because of the diffraction-limited optical resolution, we consistently produced $>1 \%$ biaxial strain by scaling the trap diameter and depth proportionally. We note such strain profiles define an approximately parabolic confinement potential for excitons, with the potential being zero at the edge, as detailed below in Supporting Information 7 and illustrated in Figure S7.

\section{Supporting Information 2. Additional experimental results on exciton emission for traps of various diameters}

Here we provide additional results of applying our lithographically defined strain-control method ${ }^{6}$ for the traps of various diameters, particularly for the case of 120-nm diameter not presented in the main text. In an unstrained flat area of the $\mathrm{MoSe}_{2}$ sample at $5 \mathrm{~K}$ (Figure S2c), the photoluminescence (PL) displays the typical exciton and trion peaks at 1.66 and $1.63 \mathrm{eV}$. To probe the response from strained regions of the sample in the traps, we tune the excitation photon energy to a value slightly below that of the unstrained optical gap. We then readily observe emission from the strained part of the sample in the trap, as shown in Figure S2a, b for two examples of 250-nm and 120-nm diameter traps. In addition to the expected red shift of the emission features, we also see a clear progression in the linewidths of the peaks. From no confinement to the $250-\mathrm{nm}$ to 120 -nm traps, the PL linewidth (full width at half maximum-FWHM) decreases from $~ 8.5$ to 4.0 to $1.5 \mathrm{meV}$ (Figure S2c, a, b). Despite the strainproduced energy spread in the trap, excitons evidently relax to the center of the trap before luminescing, as seen from the well-defined PL peaks in Figure S2a, b. We can thus attribute the reduced PL linewidth to progressively reduced inhomogeneous broadening accompanying increased exciton localization. 


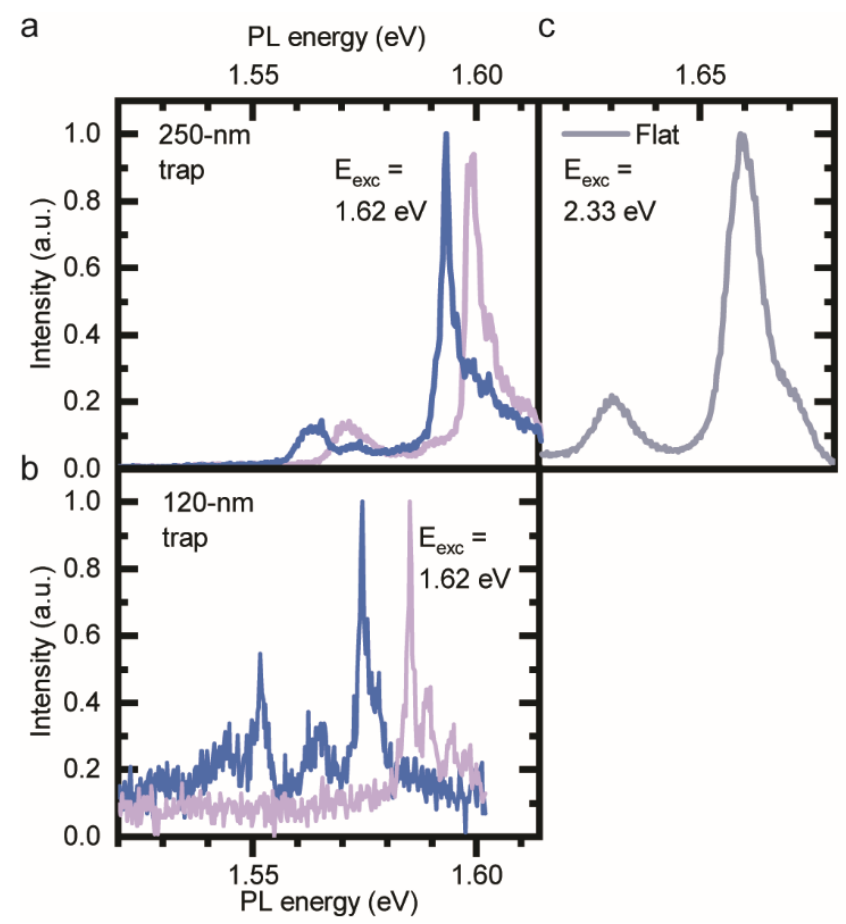

Figure S2. Photoluminescence spectra for unconfined excitons and traps to different diameters. (a) PL spectra for 250-nm and (b) $120-\mathrm{nm}$ diameter traps at a temperature of $5 \mathrm{~K}$ with an excitation photon energy $(1.62 \mathrm{eV})$ lying below the unstrained monolayer optical gap. The spectra show progressive line narrowing with greater exciton confinement. The two traces are representative results for measurements from different traps of the same diameter. In (a), the high-energy tail is attributed to luminescence from less-strained edge regions; excitons excited there do not reach the center of the trap before luminescing. (c) Reference PL spectrum (also at $5 \mathrm{~K}$ ) excited above the unstrained monolayer bandgap (at $2.33 \mathrm{eV}$ ) from a flat region of the sample, showing exciton and trion peaks at 1.66 and $1.63 \mathrm{eV}$, respectively. Note the reduced linewidths in (a), (b) vs (c).

\section{Supporting Information 3. Spectral wandering of exciton emission}

The trapped excitons investigated in this manuscript exhibited spectral wandering over time. Representative results in Figure S3a exhibit the characteristic wandering in peak position of $\sim 1-2 \mathrm{meV}$ on a time scale of an hour. We attribute this effect to photodoping of the thermal oxide substrate and the possible presence of additional two-level systems near the QEs. The spectral wandering of the QEs was found to be more pronounced at higher laser excitation intensity. The observed spectra wandering causes the measured $g^{(2)}(0)$ values to increase whenever the main peak drifts away from the $0.5-\mathrm{meV}$ detection bandwidth. To mitigate this effect, we kept the excitation power low $(<30$ $\mathrm{nW}$ ). We also measured the QEs' full spectra to center our monochromator prior to starting every $\sim 60$-minute-long TTTR integration, and used the spectra to exclude integration rounds where the QEs wandered significantly, as illustrated in Figure S3a. 

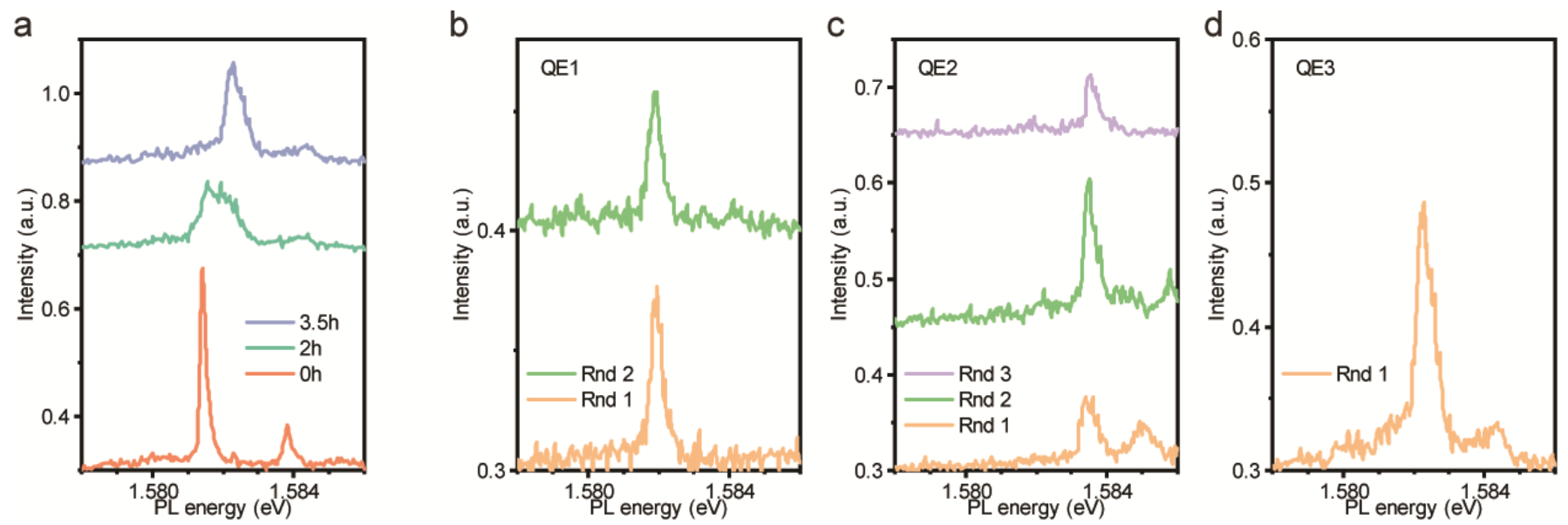

Figure S3. Spectral wandering of QEs in $\mathrm{MoSe}_{2}$. (a) QE3 exhibited spectral wandering under $1.62 \mathrm{eV}$ laser excitation at 1.8 $\mathrm{K}$. The spectra were obtained after the QE was exposed to light for 0,2 , and 3.5 hours. In this case, as significant spectral wandering occurred during 0-3.5 h, TTTR integration during this time was excluded in subsequent analysis of $g^{(2)}$ correlation data. (b), (c), (d) Spectra measured prior to integration rounds included in the analysis of $g^{(2)}$ correlation data, showing consistent peak positions between rounds.

\section{Supporting Information 4. Analysis of $g^{(2)}$ correlation data}

Here we provide details of our analysis of the $g^{(2)}$ correlation measurements. The data were collected using integration over time windows synchronized with the repetition rate of excitation from the modelocked laser. To calibrate the setup, we recorded signals generated directly from pulses of the excitation laser, as shown in the upper panel of Figure S4. The individual peaks, with a FWHM of $\sim 1.2 \mathrm{~ns}$, correspond to the instrument response function for the single-photon counting modules (SPCMs). We use this information to define the window (blue-shaded area in Figure S4) over which to integrate the coincidence counts. Representative raw data for coincidence counts from QE3 are shown in the lower panel of Figure S4. From the calibration data for laser excitation, we observe anomalously long tails for the coincidence peaks at time delays of $\pm 39.6 \mathrm{~ns}$, which we ascribe to the after-pulsing of our SPCMs. To avoid underestimating the $g^{(2)}$ values, which could occur if the average of the integrated coincidence counts were artificially high, we did not include data for time delays of $\pm 39.6 \mathrm{~ns}$ in our $g^{(2)}$ calculation. The resulting coincidence histogram for QE1 is presented in the main text. Histograms for the corresponding $g^{(2)}$ analysis for QE2 and QE3 are shown here in Figure S5.

Variations of the integrated coincidence counts over time delays followed Poissonian statistics. Explicitly, for the integrated coincidence counts of QE1-3, the average of the eight peaks adjacent to the time-zero peak are 21, 24.9, 19.1, respectively, yielding their square roots as 4.58, 4.99, and 4.37. The standard deviations calculated among the eight adjacent peaks are 4.06, 4.62, and 4.54, respectively, approaching the square roots of the averages.

We noted above the possibility of spectral wandering of the QEs' photon energy. If this energy wanders outside the detection bandwidth during integration of the coincidence measurements, then the recorded count rate is no longer dominated by the QE count rate, but rather by the count rate of background emission and detector dark current. Therefore, in our analysis, we set a threshold for the photon count rate. When the count rate dropped below this threshold, the data were not included in the coincidence calculation. For QE3, the threshold was set at 70-80\% of the maximum count rate. The high threshold led to fewer coincidences in each integration and the corresponding statistical uncertainties are taken into account in our reported $g^{(2)}(0)$ values. 


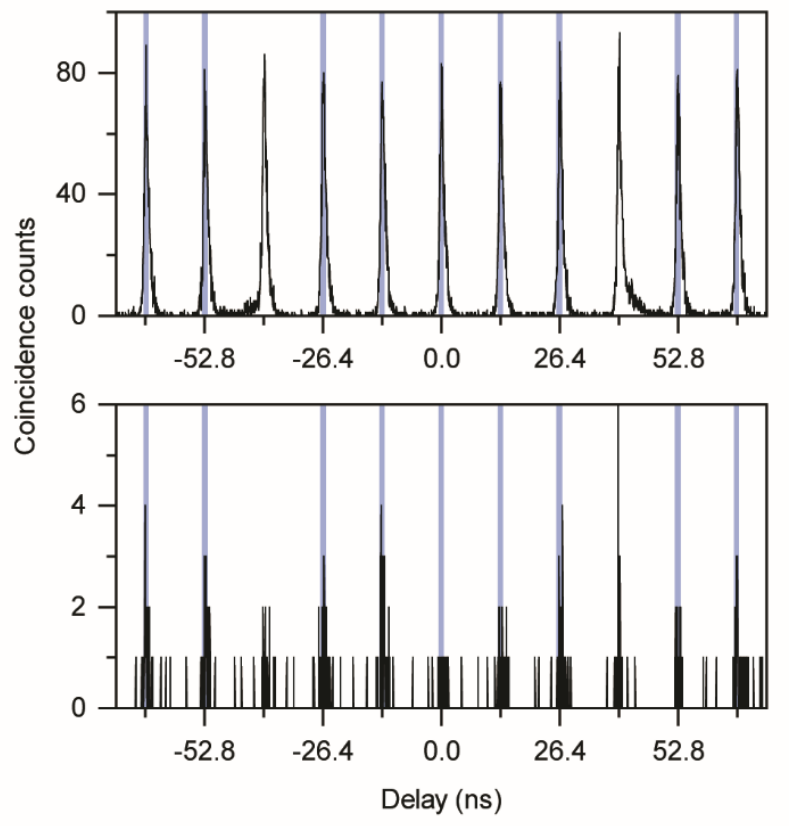

Figure S4. Photon coincidences (unintegrated) versus time of the calibration laser (top) and QE3 (bottom). The dip of QE3 at zero time delay is apparent in the raw data. The 1.2-ns-wide blue-shaded areas separated by $13.2 \mathrm{~ns}$ (corresponding to the laser's $75.8 \mathrm{MHz}$ repetition rate) indicate the time windows for integrating coincidence counts at each laser pulse. Windows at $\pm 39.6 \mathrm{~ns}$ were excluded from the $g^{(2)}$ calculation, as discussed in the text.

a

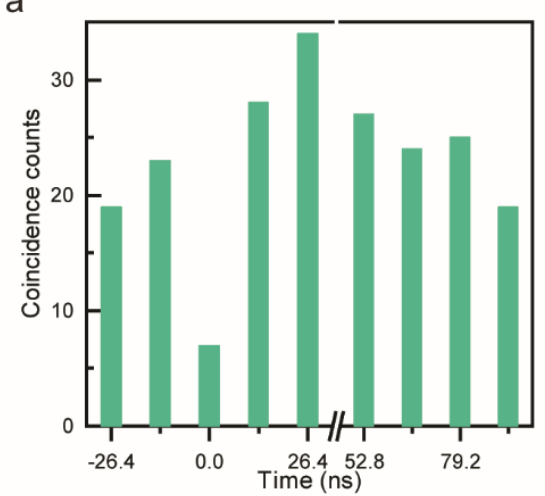

b

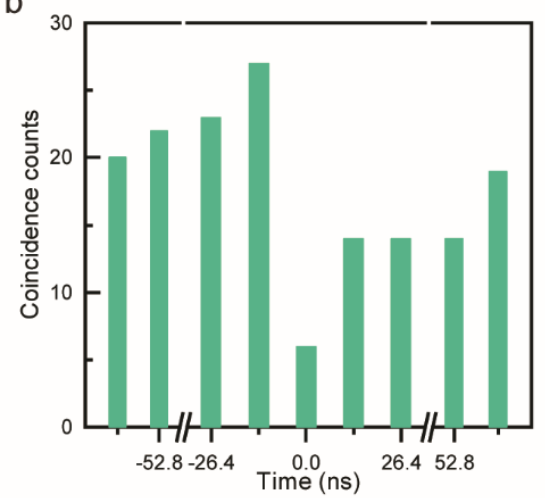

Figure S5. Integrated coincidence counts from HBT measurements. (a) QE2 with $g^{(2)}(0)$ of $0.28 \pm 0.12$. (b) QE3 with $g^{(2)}(0)$ of $0.31 \pm 0.15$.

\section{Supporting Information 5. Additional QE magneto-optical and polarization measurements}

We present additional magneto-optical and polarization measurements of the QEs in Figure S6. The measured $g$ factors for the split of the exciton emission into two lines under a perpendicular magnetic field were observed to be $3.91 \pm 0.27$ and $4.17 \pm 0.05$, respectively, for QE1 and QE3. These values of $g \approx 4$ are consistent with previous reports ${ }^{7,8}$ for free excitons in $\mathrm{MoSe}_{2}$ and further confirm our assignment of the QEs as confined spin-allowed bright excitons. On the other hand, when excited with $\sigma^{+}$polarization, the QEs showed less than $7 \%$ of circular PL 
polarization (Figure S6e). This observation is also consistent with previous measurements for free excitons in $\mathrm{MoSe}_{2}{ }^{9,10}$, an effect attributed to the rapid relaxation of the valley polarization on the light emission process ${ }^{9}$.
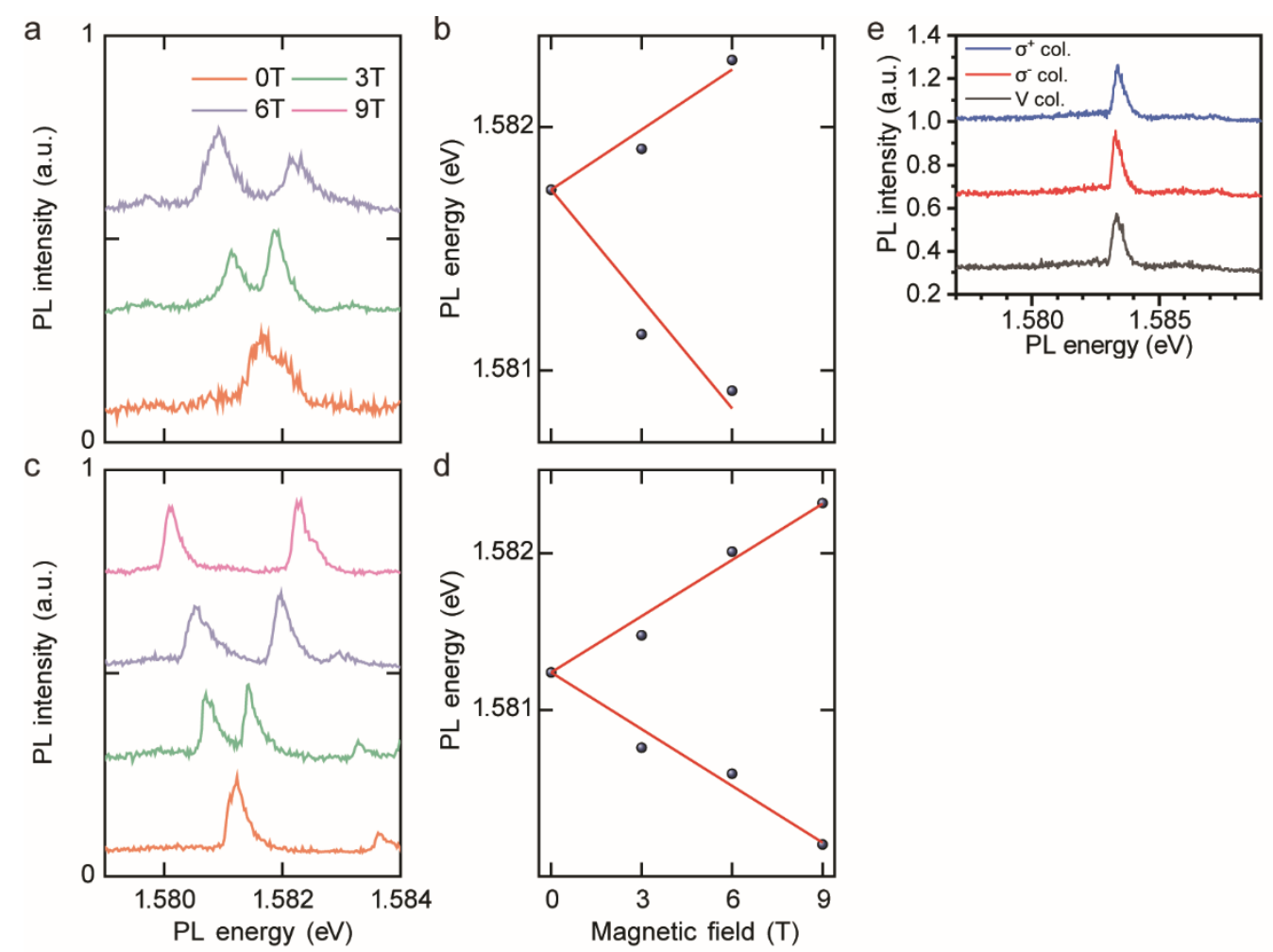

Figure S6. Additional magneto-optical and polarization measurements of the QEs. (a) Spectra of QE1 as a function of the applied magnetic field applied perpendicular to the sample. (b) Extracted PL energies for the QE1 doublet feature, fitted linearly (red line) with respect to the magnetic field to yield a $g$ factor of 3.91 \pm 0.27 . The deviation of the peak energies from the linear fit are attributed to spectral wandering, as the two peaks at a given field tracked one another. (c), (d), As above, but for another peak spatially and spectrally close to QE3, with a $g$ factor of 4.17 \pm 0.05 . (e) Polarized PL spectra from QE2 when the $1.62 \mathrm{eV}$ laser was $\sigma^{+}$-polarized. The observed low degree of circular polarization (less than $7 \%$ ) is consistent with previous measurements for free excitons in $\mathrm{MoSe}_{2}{ }^{9,10}$, possibly due to an ultrafast valley polarization relaxation dominating the relatively slow PL decay ${ }^{9}$. All measurements were performed at $1.8 \mathrm{~K}$.

\section{Supporting Information 6. Characteristics of WSe 2 quantum emitters}

$\mathrm{WSe}_{2}$ is the basis of the first-reported and most-studied QEs in an atomically thin semiconductor. To better differentiate them from the $\mathrm{MoSe}_{2}$ QEs we have developed in this work, we summarize some differences here. Notable characteristics include the nature of the excitonic transitions (i.e., dark versus bright), their radiative rates, and their Zeeman shifts ( $g$ factors) under perpendicular magnetic fields.

Important clues to the origins of emitters can be gained from knowledge of the $g$ factors in the different material systems. Bright excitons in $\mathrm{WSe}_{2}{ }^{11}$ have $g$ values close to 4, as also observed in our $\mathrm{MoSe}_{2}$ QEs. However, WSe QEs have $g$ factors that deviate strongly from 4 (and the discussion below identifies them as dark excitons). The $\mathrm{WSe}_{2}$ QEs reported to date can be broadly divided into two categories, according to their $g$ factors. The first type exhibits anomalously large $g$ factors - reported in the range of 8-10 ${ }^{12-17}, 7.2^{18}$ and $6.3^{19}$ - that are close to those of free dark excitons in $\mathrm{WSe}_{2}{ }^{20,21}$. Quantitative theoretical analysis ${ }^{20}$ shows that such QEs inherit their $g$ factors largely from host materials, despite their wavefunction hybridization with atomic defect states. The second type has a 
small $^{22}(\sim 2)$ or near-zero $g$ factor ${ }^{22,23}$. The first-reported ${ }^{12-15} \mathrm{WSe}_{2} \mathrm{QEs}$, and sizable majority of subsequently reported ones, belong to the first type, which we consider representative. This conclusion has also generally been reached in literature reports ${ }^{20,21}$ in which authors have not mentioned the second type of emitters and have associated $\mathrm{WSe}_{2} \mathrm{QEs}$ with dark exciton states.

At zero magnetic field the $\mathrm{WSe}_{2} \mathrm{QEs}$ emit cross-linearly polarized spectral doublets split by 0.2 to $0.9 \mathrm{meV}^{12,13,15,24,25}$. This is an indication of broken rotational symmetry that would arise from asymmetry in the confinement potential on a length scale comparable to or less than the $1 \mathrm{~nm}^{20,26}$ exciton Bohr radius.

Another significant characteristic of $\mathrm{WSe}_{2} \mathrm{QEs}$ is their PL decay rate $R_{\mathrm{PL}}$, typically measured to be $\sim 0.6 \mathrm{~ns}^{-1}$ or lower ${ }^{12,13,19,24,25,27}$ at cryogenic temperature. The more fundamental quantity, however, is the radiative rate $R_{\text {rad }}$. It can be derived if the photoluminescence quantum yield $(\mathrm{QY})$ is known using the relation $R_{\mathrm{rad}}=\mathrm{QY} \cdot R_{\mathrm{PL}}$. Quantum-yield measurements of QEs are challenging, and subject to large uncertainty, but literature reports ${ }^{19}$ put the radiative rate of $\mathrm{WSe}_{2} \mathrm{QEs}$ at about $0.01 \mathrm{~ns}^{-1}$.

Given its importance, we describe how $R_{\text {rad }} \sim 0.01 \mathrm{~ns}^{-1}$ was determined: In crystals prepared by chemical vapor transport (CVT) or flux growth, respectively, the $\mathrm{WSe}_{2}$ QEs have average measured PL decay rates of 0.25 or 0.07 $\mathrm{ns}^{-1}$, and measured QY of $1.5 \%$ or $16.5 \%{ }^{19}$. These figures result in radiative rates of 0.004 or $0.012 \mathrm{~ns}^{-1}$, respectively, or on the order of $0.01 \mathrm{~ns}^{-1}$. Actually, the $0.004-\mathrm{ns}^{-1}$ rate is more representative of most reported WSe $\mathrm{QEs}^{12-15,24,25,27}$, since the high-quality flux-grown crystals were demonstrated only very recently ${ }^{19,28}$ and were not widely available.

The low radiative rate of $\mathrm{WSe}_{2} \mathrm{QEs}$ is also consistent with their origin as nominally spin-forbidden dark-exciton transitions ${ }^{20,21}$. By way of comparison, $\mathrm{MoSe}_{2}$ QEs have much higher estimated radiative rates, as discussed in detail in Supporting Information 10 below.

\section{Supporting Information 7. Modeling of exciton-localization effects in traps}

Localization of excitons in strain-induced traps influences their center-of-mass motion and linewidth. If more than one exciton is confined in such a well, their interaction strength also depends on the trap's properties. Here we examine the influence of the trap potential on these characteristics to determine when we expect to enter the regime of quantum emission.

(1) Center-of-mass motion of excitons in the strain-induced trap

The spatial profile of traps in the $\mathrm{MoSe}_{2}$ monolayer was arranged to produce an expected well depth of $E_{S} \sim 70 \mathrm{meV}$ for all trap diameters, as confirmed by the observed shift in emission exciton energy from the QEs compared with that from unstrained regions on the monolayer. Since the extent of exciton center-of-mass wavefunction is small compared with the trap diameter (see below), the excitons experience nearly the maximum degree of strain tuning.

To determine shape of the expected trap potential, we approximate the $\mathrm{MoSe}_{2}$ strain distribution as a parabola that vanishes at the trap edge. The approximated strain is an effective isotropic strain taken as the average of the radial and azimuthal strains. The approximated distribution is justified by the extended Hencky solution ${ }^{3}$ for a clamped membrane under pressure described above in Supporting Information 1. We then calculate the influence on the exciton energy using the $54 \mathrm{meV} / \%$ strain tuning rate ${ }^{29}$. We plot the resulting strain-induced exciton confinement potential in Figure S7a. 

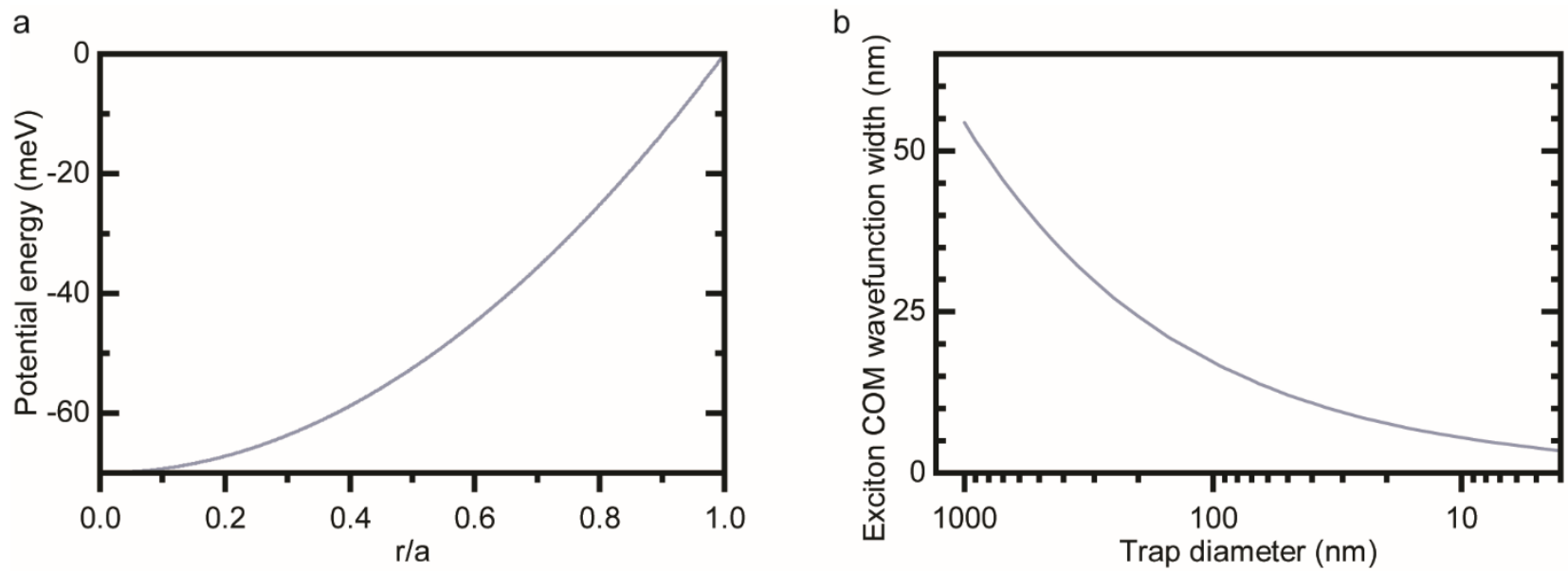

Figure S7. Modelling of strain confinement. (a) Strain potential profile modeled according to an approximate parabolic strain distribution that vanishes at the clamp edge $(r / a \rightarrow 1)$. The approximation is justified by the extended Hencky solution ${ }^{3}$ (Supporting Information 1). (b) Exciton $\left(1 / e^{2}\right) \mathrm{COM}$ wavefunction width subsequently used to estimate exciton interaction. The exciton width is proportional to the square root of the trap diameter, as expected for a constant-depth harmonic potential (see below).

We examine exciton confinement in the weakly localized regime where we can neglect modification of the internal exciton wavefunction. We can thus focus on the exciton center-of-mass (COM) motion, which we describe quantum mechanically. In Figure S8, we plot the quantization energy for the COM motion of the exciton, which we take as the difference in energy between the first-excited and ground states, as a function of the trap diameter for a constant well depth of $70 \mathrm{meV}$. The quantization energy scales with the width of the trap as $1 / a$, as expected for a quadratic potential (harmonic oscillator) of constant depth. We show in Figure S7b the corresponding $\left(1 / e^{2}\right)$ width for the COM motion of ground-state exciton, which scales with trap size as $a^{1 / 2}$. In these calculations, we have used an exciton mass of $M=m_{e}+m_{h}=0.5 m_{0}$. Note that under thermal equilibrium at our experimental temperature of $T=$ $1.8 \mathrm{~K}$ (thermal energy of $0.16 \mathrm{meV}$ ), we expect the exciton to be in its translational ground state for diameters below $\sim 1 \mu \mathrm{m}$.

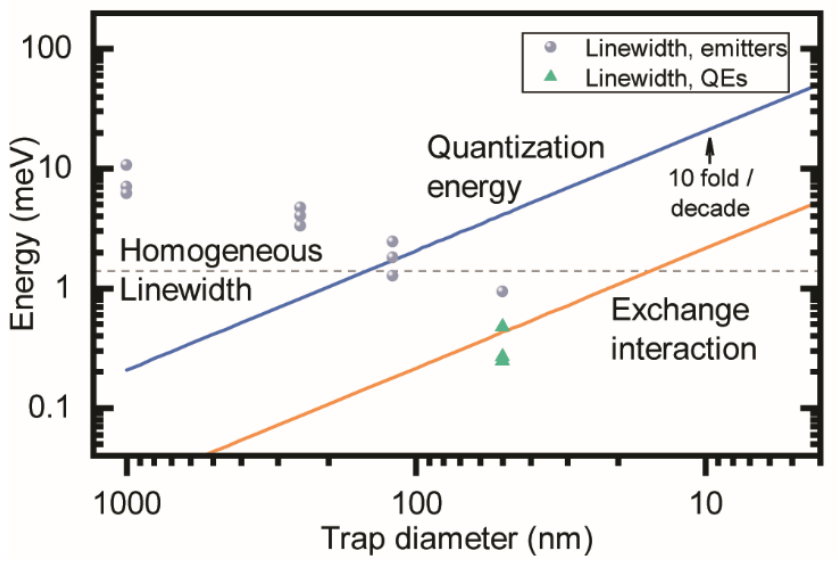

Figure S8. Exciton localization effects. Experimentally measured linewidths (in FWHM, gray circles and green triangles, obtained from Figure 1b, 2c, Figure S2) reduced in smaller trap confinements, eventually drops below the homogeneous linewidth $\left(1.4 \mathrm{meV}^{30}\right)$ represented as the dashed line. However, the theoretical exchange interaction energy is barely comparable to the experimental linewidth, even in the smallest 50-nm trap, leaving open the interpretation that our observed 
QEs may benefit from additional potential fluctuations (Supporting Information 8). On the other hand, when the exchange interaction energy exceeds $1 \mathrm{meV}$ in 20-nm traps, the corresponding exciton width of $8 \mathrm{~nm}$ (Figure S7) would yield a radiative rate of $3 \mathrm{~ns}^{-1}$ (Fig. 4), still greater than $1 \mathrm{~ns}^{-1}$. Details of the calculations appear in Supporting Information 7.

(2) Exciton-exciton interactions in a trap

When two excitons are present simultaneously in the trap, single-photon emission and detection is possible if the exciton-exciton interaction shifts the transition energy away from that of two noninteracting excitons. To estimate the exciton interaction energy we adopt the treatment by $\mathrm{Yu}$ et $a l^{31}{ }^{31}$, which identifies the exchange interaction as the strongest one between excitons with the same spin indices:

$$
U_{\mathrm{ex}} \approx \frac{1}{2 \pi}\left(\frac{a_{B}}{w}\right)^{2} V_{\mathrm{ex}}
$$

Here $V_{e x} \sim E_{b}$, the exciton binding energy; $a_{B}$ is the exciton Bohr radius; and $w$ is the $1 / e^{2}$ width of the exciton $\mathrm{COM}$ wavefunction in real space. Using parameters of $a_{B}=1 \mathrm{~nm}, E_{b}=0.4 \mathrm{eV}$, and our calculated exciton wavefunction COM widths (Figure S7b,) we obtain an estimate for the exciton-exciton interaction as a function of well diameter ( $2 a)$ as shown in Figure S8. For two excitons with opposite spin indices, the exchange interaction is absent, so a dipole-dipole interaction dominates. But this dipolar interaction is weaker than the $U_{\text {ex }}$ calculated above $^{31}$, which is already smaller than the experimental linewidth shown in Figure S8. This suggests that even in a best-case estimate, the overall exciton interaction in the 50-nm trap is not sufficient to account for the observed quantum emission. Thus, additional extrinsic effects may play a role in our observed QEs (see Supporting Information 8).

\section{Supporting Information 8. Additional contributions towards exciton localization in $\mathrm{MoSe}_{2}$ quantum emitters}

We estimated that the exciton interaction energy in the 50-nm trap was not sufficient to dominate the experimental linewidths (Supporting Information 7) and allow us to reject emission when two excitons are present simultaneously. This suggests that our QEs may benefit from potential fluctuations arising from variations in the local topography or other substrate characteristics, such as local trapped charges ${ }^{32}$. Such fluctuations might induce attractive exciton potentials $\sim 10-\mathrm{meV}$ deep on a 10-nm (or smaller) spatial scale. The resulting bound excitons would be expected to retain in large measure the spin configuration and $g$ factor of the intrinsic band structure of the host material. The tighter confinement would, however, force the excitons into more intimate contact, increasing their interaction strength $U_{\mathrm{ex}}$ beyond the experimental linewidths. Quantum emission would occur in a (strain plus fluctuation) confinement that contained one well or multiple spectrally separated wells.

\section{Supporting Information 9. Distinguishing between neutral excitons and charged excitons}

We provide additional considerations in determining whether our QEs are based on neutral excitons or on trions ("charged excitons"). Emission features from both species are evident in our spectra (Figure 1 and Figure S2). Their transition energies are not so widely separated as to allow an unambiguous assignment.

Based on our good (but not perfect) control over the strain tuning, the case for neutral excitons is strong. The 1.58 $\mathrm{eV}$ photon energy for QEs in our 50-nm traps was close to that of the neutral-exciton in 250-nm traps (Figure 1b). The $1.58 \mathrm{eV}$ energy also meets expectations based on the $1.66 \mathrm{eV}$ zero-strain starting point (Figure 1c), the 54 meV/\% strain tuning rate, and the target $\sim 1.5 \%$ biaxial strain. However, we cannot completely rule out the possibility that fabrication errors led to reduced strain, that the spectral shift was only $\sim 0.05 \mathrm{eV}$ instead of $0.08 \mathrm{eV}$, and that the $1.58 \mathrm{eV}$ feature arises from bound trions.

We thus consider other experimental means to distinguish between neutral excitons and trions. Electrical gating (to modulate the concentration of free carriers) is a standard test for the presence of charged versus neutral excitons, 
the relative amplitudes of their spectral peaks responding to changes in the gate bias. Unfortunately, our sample did not provide this capability.

Purely optical measurements can also allow the identification a pair of related spectral peaks - the lower energy peak would then be associated with trions. Performing such optical measurements, including photoluminescence excitation and photon cross-correlation, would however require the trion binding energy to be known a priori. Recently, the trion binding energies of $\mathrm{WSe}_{2}$ QEs have been reported ${ }^{16,33,34}$ with disparate values: $\sim 10 \mathrm{meV}$ in the former two papers ${ }^{16,33}$ and $>20 \mathrm{meV}$ in the latter ${ }^{34}$. The influence of localization on trion binding energies remains an open question, making such identification of correlated spectral peaks difficult.

Nonetheless, even if trions were responsible for our QEs, we note that $\mathrm{MoSe}_{2}$ QEs still hold better promise than the $\mathrm{WSe}_{2}$ counterparts for quantum valleytronics. While the unresolvable zero-field splittings of our $\mathrm{MoSe}_{2} \mathrm{QEs} \mathrm{might}$ be simply due to the protection of Kramers' theorem, there was no evidence that localized neutral excitons in $\mathrm{MoSe}_{2}$ have to show broken valley degeneracy, in contrast to the $\mathrm{WSe}_{2}$ QEs that repeatedly exhibit spectral doublets ${ }^{12,13,15,24,25}$.

\section{Supporting Information 10. Radiative rates of localized excitons}

Radiative rate is an important property of a quantum emitter, since it establishes the maximum photon-emission rate. For an exciton in a semiconductor nanostructure, the radiative rate depends on the degree of spatial confinement. Qualitatively, this behavior can be understood by noting that a decrease in radiative rate is expected once the exciton is confined to a spatial extent on the order of the wavelength of light. At that point, Fourier components of the exciton center-of-mass (COM) wavefunction begin to include wavevectors that lie outside the light cone and do not contribute to far-field radiation. Here we describe our application of existing theoretical treatments to our case of harmonic lateral confinement in the $\mathrm{MoSe}_{2}$ monolayer which have already been summarized in Figure 4 of the main text.

\section{Theoretical considerations}

The reduction of radiative rate with degree of spatial confinement of an exciton has been calculated in various papers $^{35-38}$. We follow the theory by Sugawara ${ }^{38}$, noting that the theory by Citrin ${ }^{35,36}$ produces the same result. Sugawara's theory was originally developed for localized excitons in quantum wells, which treats the exciton COM motion within a harmonic confinement potential. The resulting COM wavefunction is expanded into a Fourier series in momentum space where only the components within the light cone contribute to radiative decay. The radiative rate $R$ in Figure 4 was calculated using a relation in Sugawara's paper ${ }^{38}$ (their Eqn. (42)) under the assumption that the COM motion of the exciton corresponds to its ground translational state:

$$
R(\beta)=\frac{e^{2} \beta^{2}}{m_{0} \varepsilon_{0} c n} \int_{0}^{\kappa_{\mathrm{ex}}} \exp \left(-\beta^{2} q^{2} / 2\right)\left[f_{\|} \eta_{1}(q)+f_{\perp} \eta_{2}(q)\right] q d q .
$$

Here the parameter $\beta=\sqrt{2 \hbar / m_{\mathrm{ex}} \omega}$ is the $1 / e^{2}$ radius of the exciton COM wavefunction as determined by the eigenfrequency $\omega$ of the harmonic potential and the exciton total mass $m_{\mathrm{ex}} ; f_{\|}$and $f_{\perp}$ are the oscillator strengths for emission polarized parallel and perpendicular to the 2D layer, respectively; and $\eta_{1}(q)$ and $\eta_{2}(q)$ describe the momentum dependence of radiative decay. The integration region is bounded by the light-wavevector in the material, $\kappa_{\mathrm{ex}}=E_{\mathrm{ex}} n / \hbar c$, where $E_{\mathrm{ex}}$ is the exciton and $n$ is the refractive index of the surrounding media. Other symbols have their usual meaning, with $m_{0}$ denoting the electron mass.

To adapt the theory ${ }^{38}$ for the localized excitons in the $\mathrm{MoSe}_{2}$ monolayer, we let the quantum well thickness $L_{z}$ go to zero so that the exciton motion become strictly two dimensional. Emission from the bright exciton in $\mathrm{MoSe}_{2}$ is polarized in-plane, so $f_{\perp}=0$. We determine $f_{\|}$by requiring that $R(\beta \rightarrow \infty)=R_{0}$ reproduces the free-exciton radiative rate. We take $R_{0}=2 \mathrm{ps}^{-1}$ based on an experimental low-temperature homogeneous linewidth of $1.4 \mathrm{meV}^{30}$. 
Similar THz intrinsic radiative rates have been inferred ${ }^{39}$ in other experimental studies and calculated theoretically ${ }^{40}$. We take $n=\left(1 / 2\left(1 / n_{\text {air }}+1 / n_{\mathrm{Si}}\right)\right)^{-1}$ to account for the radiation into two half-spaces of different optical media ${ }^{38}$.

We have calculated the radiative rates of excitons confined down to a 4-nm width, which yields the slowest predicted radiative rate of $1 \mathrm{~ns}^{-1}$. Still tighter exciton confinement is implausible in view of our experimental findings. First, the predicted zero-point energy of the exciton in a 4-nm well already exceeds $30 \mathrm{meV}$, but we do not observe a significant spectral blue shift, in the measured PL energies of the QEs (Figure 2c) compared to the 250-nm trap (Figure 1b). Further, for confinement tighter than 4-nm, we would anticipate changes to the internal structure of the exciton, which has a diameter of $\sim 2 \mathrm{~nm}$. We see no experimental signature of such an effect, for example, in the measured $g$-factor of the QEs compared to free excitons ${ }^{7,8}$.

We note that for our potential well, the energy level spacing of the COM states will exceed $1 \mathrm{meV}$ for $\beta<20 \mathrm{~nm}$. In this regime, we are therefore well justified in considering only occupancy of the ground state for the COM motion at cryogenic temperatures, e.g., $T=4 \mathrm{~K}=0.35 \mathrm{meV} / k_{B}$.

(2) Experimental considerations

The radiative rate of an emitter can be inferred from its measured homogeneous (transform-limited) linewidth or its photoluminescence decay rate and quantum yield (QY). The latter procedure is standard for QEs, which often do not exhibit transform-limited linewidths because their relatively low radiative rates compete poorly with other decoherence processes. The QY is typically measured by comparing the number of emitted photons with the number of excitation pulses, which are made sufficiently intense to ensure that emission occurs for every excitation pulse. The number of emitted photons in such measurements quantitively indicates brightness of QEs and is proportional to QY.

We could not follow the standard procedure to measure the QY of $\mathrm{MoSe}_{2}$ QEs due to the aforementioned spectral wandering effect (Supporting Information 3). While the QE did generate more photons with increased excitation power, its emission peak also wandered and broadened, possibly due to a combination of the photodoping effect associated with thermal oxide substrates and the presence of additional two-level systems near the QE. Consequently, at high excitation levels, we could not isolate single $\mathrm{MoSe}_{2}$ QEs to measure their QY.

Because the $\mathrm{MoSe}_{2}$ QE pump transitions were not saturated, the photon-counting procedure for measuring QY allowed us only to obtain a lower bound - the number of detected photons is known, but for the number of excitons created we have only the upper bound of the number of excitation pulses. Using the measured total count rate of $2000 / \mathrm{sec}$ from the QE registered on SPCMs, the overall system efficiency of $\sim 1 \%$ of our setup, and the laser repetition rate of $75.8 \mathrm{MHz}$, we arrived at a lower bound on the quantum yield of $(2000 \mathrm{~Hz}) /(0.01)(75.8 \mathrm{MHz}) \sim$ $0.3 \%$. We believe that this is a significant underestimate of the true quantum yield. It is possible to refine the QY estimate by calculating the number of pump photons absorbed (and excitons produced) in the QE. For such an estimate, one would need to model the pump-laser field distribution, the monolayer absorption (and the influence of strain on it), and the exciton diffusion into the QE from surrounding regions. Given the uncertainties in these quantities, we have not attempted to follow this route.

\section{Supporting Information 11. Temperature-dependent photoluminescence from $\mathrm{MoSe}_{2}$ quantum emitters}

We present temperature-dependent PL spectra from the three QEs at $\mathrm{T}=2,10,30 \mathrm{~K}$. As shown in Figure S9, the QE peaks broaden with increasing temperature. Despite slight variations in peak positions that we attribute to spectral wandering, the peaks do not exhibit any significant systematic shift with temperature increases from 2 to $30 \mathrm{~K}$ (corresponding to a thermal energy increase from 0.2 to $2.6 \mathrm{meV}$ ). The lack of a shift in emission energy despite the meV-scale increase in thermal energy in the system is consistent with our estimate of the exciton quantization energy of at least $4 \mathrm{meV}$ in a $50-\mathrm{nm}$ strain trap. 

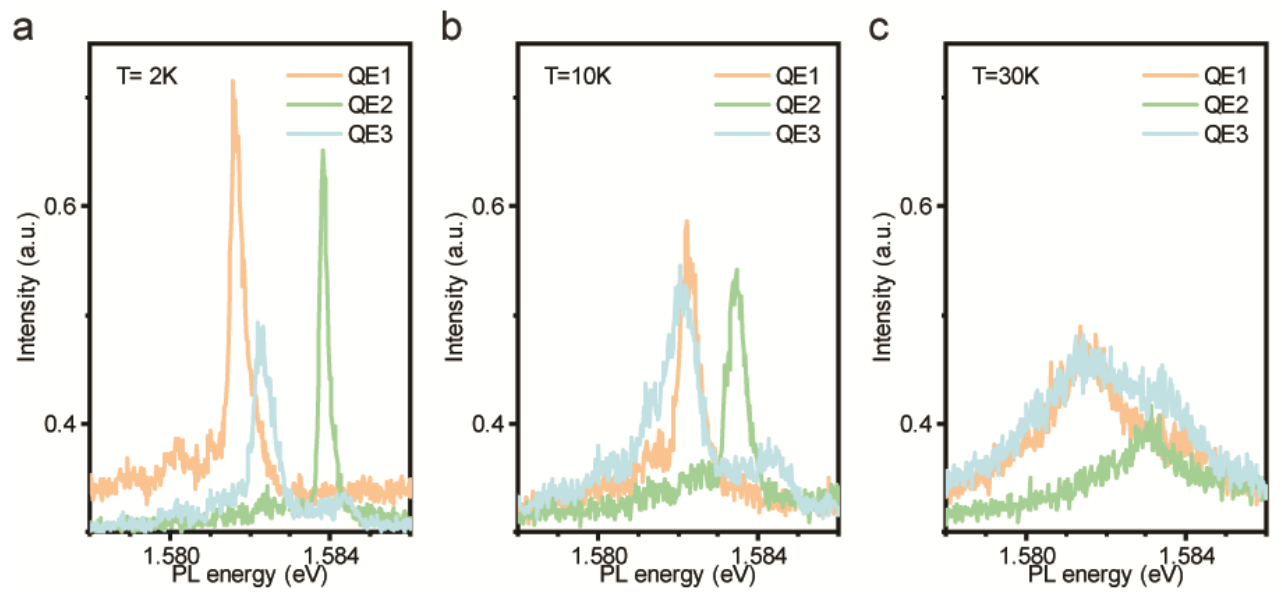

Figure S9. Temperature-dependent PL spectra for QEs at $\mathrm{T}=2,10,30 \mathrm{~K}$. The $\mathrm{QE}$ emission energies remain largely unchanged from 2 to $30 \mathrm{~K}$, consistent with our estimate of the exciton quantization energy of $\sim 4 \mathrm{meV}$ in a 50-nm strain trap (Figure S8). 


\section{Supporting references}

1. Hencky, H. Über den spannungszustand in kreisrunden platten mit verschwindender biegungssteiflgkeit. Z. fur Math. und Phys. 63, 311-317 (1915).

2. Koenig, S. P., Boddeti, N. G., Dunn, M. L. \& Bunch, J. S. Ultrastrong adhesion of graphene membranes. Nat. Nanotechnol. 6, 543-546 (2011).

3. Kitt, A. L. et al. How graphene slides: Measurement and theory of strain-dependent frictional forces between graphene and SiO2. Nano Lett. 13, 2605-2610 (2013).

4. Kang, J., Tongay, S., Zhou, J., Li, J. \& Wu, J. Band offsets and heterostructures of two-dimensional semiconductors. Appl. Phys. Lett. 102, 012111 (2013).

5. Yang, Y. et al. Brittle Fracture of 2D MoSe 2. Adv. Mater. 29, 1604201 (2017).

6. Yu, L. et al. Lithographically-defined strain control in atomically-thin semiconductors. Prep.

7. Li, Y. et al. Valley Splitting and Polarization by the Zeeman Effect in Monolayer MoSe2. Phys. Rev. Lett. 113, 266804 (2014).

8. MacNeill, D. et al. Breaking of Valley Degeneracy by Magnetic Field in Monolayer MoSe2. Phys. Rev. Lett. 114, 037401 (2015).

9. Wang, G. et al. Polarization and time-resolved photoluminescence spectroscopy of excitons in MoSe2 monolayers. Appl. Phys. Lett. 106, 112101 (2015).

10. Glazov, M. M. et al. Spin and valley dynamics of excitons in transition metal dichalcogenide monolayers. Phys. Status Solidi Basic Res. 252, 2349-2362 (2015).

11. Srivastava, A. et al. Valley Zeeman effect in elementary optical excitations of monolayer WSe2. Nat. Phys. 11, 141147 (2015).

12. Srivastava, A. et al. Optically active quantum dots in monolayer WSe2. Nat. Nanotechnol. 10, 491-496 (2015).

13. He, Y.-M. et al. Single quantum emitters in monolayer semiconductors. Nat. Nanotechnol. 10, 497-502 (2015).

14. Koperski, M. et al. Single photon emitters in exfoliated WSe2 structures. Nat. Nanotechnol. 10, 503-506 (2015).

15. Chakraborty, C., Kinnischtzke, L., Goodfellow, K. M., Beams, R. \& Vamivakas, A. N. Voltage-controlled quantum light from an atomically thin semiconductor. Nat. Nanotechnol. 10, 507-511 (2015).

16. Lu, X. et al. Optical initialization of a single spin-valley in charged WSe2 quantum dots. Nat. Nanotechnol. 14, 426431 (2019).

17. Chakraborty, C. et al. Quantum-Confined Stark Effect of Individual Defects in a van der Waals Heterostructure. Nano Lett. 17, 2253-2258 (2017).

18. Kumar, S., Kaczmarczyk, A. \& Gerardot, B. D. Strain-Induced Spatial and Spectral Isolation of Quantum Emitters in Mono- and Bilayer WSe 2. Nano Lett. 15, 7567-7573 (2015).

19. Luo, Y. et al. Deterministic coupling of site-controlled quantum emitters in monolayer WSe2 to plasmonic nanocavities. Nat. Nanotechnol. 13, 1137-1142 (2018).

20. Linhart, L. et al. Localized Intervalley Defect Excitons as Single-Photon Emitters in WSe 2. Phys. Rev. Lett. 123, 146401 (2019).

21. Koperski, M. et al. Orbital, spin and valley contributions to Zeeman splitting of excitonic resonances in MoSe2, WSe2 and WS2 Monolayers. 2D Mater. 6, 015001 (2018).

22. Dang, J. et al. Identifying defect-related quantum emitters in monolayer WSe2. npj 2D Mater. Appl. 4, 2 (2020).

23. Kumar, S. et al. Resonant laser spectroscopy of localized excitons in monolayer WSe_2. Optica 3, 882 (2016). 
24. Branny, A., Kumar, S., Proux, R. \& Gerardot, B. D. Deterministic strain-induced arrays of quantum emitters in a twodimensional semiconductor. Nat. Commun. 8, 15053 (2017).

25. Palacios-Berraquero, C. et al. Large-scale quantum-emitter arrays in atomically thin semiconductors. Nat. Commun. 8 , 15093 (2017).

26. You, Y. et al. Observation of biexcitons in monolayer WSe2. Nat. Phys. 11, 477-481 (2015).

27. Tonndorf, P. et al. Single-photon emission from localized excitons in an atomically thin semiconductor. Optica 2, 347 (2015).

28. Edelberg, D. et al. Approaching the Intrinsic Limit in Transition Metal Diselenides via Point Defect Control. Nano Lett. 19, 4371-4379 (2019).

29. Island, J. O., Kuc, A., Diependaal, E. H., Zant, H. S. J. Van Der \& Heine, T. Precise and reversible band gap tuning in single-layer MoSe2 by uniaxial strain. Nanoscale 8, 2589-2593 (2015).

30. Ajayi, O. a et al. Approaching the intrinsic photoluminescence linewidth in transition metal dichalcogenide monolayers. 2D Mater. 4, 031011 (2017).

31. Yu, H., Liu, G.-B., Tang, J., Xu, X. \& Yao, W. Moiré excitons: From programmable quantum emitter arrays to spinorbit-coupled artificial lattices. Sci. Adv. 3, e1701696 (2017).

32. Zhang, Y., Brar, V. W., Girit, C., Zettl, A. \& Crommie, M. F. Origin of spatial charge inhomogeneity in graphene. Nat. Phys. 5, 722-726 (2009).

33. Chakraborty, C. et al. 3D Localized Trions in Monolayer WSe2 in a Charge Tunable van der Waals Heterostructure. Nano Lett. 18, 2859-2863 (2018).

34. Brotons-Gisbert, M. et al. Coulomb blockade in an atomically thin quantum dot coupled to a tunable Fermi reservoir. Nat. Nanotechnol. 14, 442-446 (2019).

35. Citrin, D. S. Radiative lifetimes of excitons in quantum wells: Localization and phase-coherence effects. Phys. Rev. B 47, 3832-3841 (1993).

36. Citrin, D. S. Radiative lifetimes of excitons in semiconductor quantum dots. Superlattices Microstruct. 13, 303 (1993).

37. Björk, G., Pau, S., Jacobson, J. \& Yamamoto, Y. Wannier exciton superradiance in a quantum-well microcavity. Phys. Rev. B 50, 17336-17348 (1994).

38. Sugawara, M. Theory of spontaneous-emission lifetime of Wannier excitons in mesoscopic semiconductor quantum disks. Phys. Rev. B 51, 10743-10754 (1995).

39. Dufferwiel, S. et al. Exciton-polaritons in van der Waals heterostructures embedded in tunable microcavities. Nat. Commun. 6, 8579 (2015).

40. Robert, C. et al. Exciton radiative lifetime in transition metal dichalcogenide monolayers. Phys. Rev. B 93, 205423 (2016). 